\title{
The Global Trends and Regional Differences in Incidence of Dengue Infection from 1990 to 2019: An Analysis from the Global Burden of Disease Study 2019
}

Min Du $\cdot$ Wenzhan Jing $\cdot$ Min Liu $\cdot$ Jue Liu

Received: March 31, 2021 / Accepted: May 25, 2021 / Published online: June 26, 2021

(C) The Author(s) 2021

\section{ABSTRACT}

Introduction: Dengue, as a neglected tropical disease, brings a heavy socioeconomic burden. To provide tailored global prevention strategies, we analyzed the global trends and regional differences in incidence of dengue infection from 1990 to 2019.

Methods: We obtained data on annual dengue episodes and incidence rates, which reflected the epidemic status of dengue infection from the 2019 Global Burden of Disease (GBD) Study. The changes in dengue episodes and estimated annual percentage changes (EAPCs) of the agestandardized incidence rate (ASR) were calculated to quantify the temporal trends of dengue infection.

Results: Globally, dengue ASR increased by $1.70 \%$ (95\% CI $1.62 \%-1.78 \%$ ) per year from

Min Du and Wenzhan Jing contributed equally.

Supplementary Information The online version contains supplementary material available at https:// doi.org/10.1007/s40121-021-00470-2.

M. Du · W. Jing $\cdot$ M. Liu ( $₫) \cdot$ J. Liu $(\bowtie)$

Department of Epidemiology and Biostatistics, School of Public Health, Peking University, No.38, Xueyuan Road, Haidian District, Beijing 100191, China

e-mail: liumin@bjmu.edu.cn

J. Liu

e-mail: jueliu@bjmu.edu.cn
1990 to 2011; subsequently, it decreased by $0.41 \%$ (95\% CI $0.20 \%-0.62 \%$ ) per year from 2011 to 2019. However, the global number of dengue episodes increased steadily by $85.47 \%$ from 30.67 million in 1990 to 56.88 million in 2019. Against the global trend of decreasing ASR from 2011 to 2019, an increasing trend was reported in Oceania (EAPC 11.01, 95\% CI 8.79-13.27), East Asia (EAPC 4.84, 95\% CI 2.70-7.03) and Southeast Asia (EAPC 0.38, 95\% CI 0.13-0.62). For socio-demographic index (SDI) regions, ASR continued to have an increasing trend in the middle (EAPC 0.26, 95\% CI 0.07-0.45) and high-middle (EAPC 1.70, 95\% CI 0.98-2.42) SDI regions from 2011 to 2019. In contrast to the global peak age of dengue incidence rate (10 to 25 years), the dengue incidence rate of older people ( $>65$ years) was higher than in other age groups in low and lowmiddle SDI regions. Additionally, the proportions of dengue episodes in the $>70$-year-old age group increased in 2019 (using the baseline in 1990 or 2011) in most GBD regions.

Conclusions: Global dengue episodes have increased tremendously in 3 decades. Although global dengue ASR decreased in the last decade, it is still increasing in hyperendemic regions including Oceania, East Asia and Southeast Asia, and also in the middle and high-middle SDI regions. More attention should be paid to the elderly because of the higher dengue incidence rate among them in low and low-middle SDI regions and the increased proportions of 
dengue episodes among the elderly in most GBD regions. Therefore, more efforts should be undertaken to develop targeted prevention strategies for crucial regions and older populations.

Keywords: Dengue; Incidence; Trend

\section{Key Summary Points 3}

Hyperendemic regions including Oceania, East Asia and Southeast Asia still had an increasing age-standardized incidence rate (ASR) of dengue infection in the last decade.

Both middle and high-middle SDI regions also had an increasing dengue infection ASR in the last decade.

There were higher dengue incidence rates among the elderly than in other age groups in low and low-middle SDI regions.

There have been increasing numbers of dengue episodes among the elderly in most GBD regions.

More attention should be paid to developing targeted prevention strategies for dengue infection in crucial regions and older populations.

\section{DIGITAL FEATURES}

This article is published with digital features, including a summary slide, to facilitate understanding of the article. To view digital features for this article go to https://doi.org/10.6084/ m9.figshare.14666130.

\section{INTRODUCTION}

Dengue, one of the neglected tropical diseases, is the most common mosquito-borne viral disease, with a heavy disease burden globally $[1,2]$. The World Health Organization (WHO) estimated that there were 100 million symptomatic cases a year from 2000 to 2013 [3]. It was reported that half of the global population is at risk of dengue infection [4]. Over the past 10 years, the emergence and re-emergence of dengue globally has posed great challenges for public health. Large-scale dengue outbreaks have occurred in many countries, such as the 2019 outbreaks in Nepal [5], 2012-2015 outbreaks in China [6] and 2014-2015 outbreaks in Taiwan (province of China) [7]. A predictive model speculated that populations with dengue infection will increase by 2.25 billion from 2015 to 2080 [8].

Dengue virus (DENV) belongs to the genus Flavivirus and contains four serotypes transmitted between humans including DENV 1, 2, 3 and 4 [9]. The DENV 5 serotype, which was discovered in Malaysia in 2007, follows the sylvatic cycle, unlike the other four serotypes [10]; nevertheless, it still can cause human disease [11]. Dengue virus is spread by a humanmosquito-human cycle with the mosquito Aedes aegypti as the primary vector and Aedes albopictus as a secondary vector [9]. Aedes was originally found in tropical and sub-tropical zones, but now has spread to almost all continents [12]. Non-vector transmission sometimes occurs in humans, such as through blood transfusion, organ transplantation and needle stick injuries [1]. Adolescents and adults are mostly affected by dengue in most hyperendemic areas $[1,6]$. However, according to some reported data from hospitals, dengue infection is also becoming a serious threat for older people in Thailand and China $[13,14]$. Many complex and interactive factors accelerate the occurrence of dengue infection including climate [15, 16] (rainfall, temperature and humidity, etc.), transportation [1], population density [16, 17], extreme poverty and inadequate sanitation [18], among others.

Vaccination is regarded as the most efficient strategy to control infectious disease. Starting in December 2015, the tetravalent dengue vaccine (CYD-TDV, marketed as Dengvaxia ${ }^{\circledR}$ ) was licensed in 20 countries for individuals between 9 and 45 years of age $[11,12]$. A model estimated that if this vaccine targeted seropositive recipients as recommended by $\mathrm{WHO}$, dengue 
episodes might be reduced to varying extents among different age groups and populations [19]. However, there is insufficient clinical and epidemiological evidence to approve the safety of a vaccine in humans, and more clinical trials are needed to evaluate the safety and efficacy [20-22]. Up to now, the main prevention and control strategy of dengue infection is vector control $[12,23]$. Some countries use combined control measures to reduce the possibilities of dengue outbreaks locally [24]. Recently studies have shown existing control measures have been ineffective at curbing the increasing global incidence of dengue infection, so promoting vaccination strategies for implementation and new comprehensive combined measures is crucial $[25,26]$.

To reduce the mortality and morbidity of dengue, WHO put forward the global strategy for dengue prevention and control 2012-2020 [27]. A Global Burden of Disease (GBD) study estimated the number of global dengue episodes increased to 58.4 million in 2013 [28]. However, descriptions of the latest trends and age distribution characteristics of dengue infection at the global, regional and national levels are lacking. Therefore, we analyzed the current epidemic status and temporal trends of dengue infection from 1990 to 2019 at the global, regional and national levels, using the data on the incidence of dengue infection from the 2019 GBD results [29]. The aim of this study is to provide a more comprehensive perspective to help develop global targeted strategies for dengue prevention by examining the global landscape, long-term trends and regional differences in the incidence of dengue infection.

\section{METHODS}

\section{Data Source}

The GBD study is coordinated by the Institute for Health Metrics and Evaluation at the University of Washington, USA, and consists of a systematic and scientific effort to quantify the comparative magnitude of health losses due to diseases by sex, age, region and country over time [29]. From the Global Health Data
Exchange website, established by the GBD group, we obtained data including annual dengue episodes and incidence rates of dengue infection from 1990 to 2019 by sex, age, region and country [29]. The dengue infection data covered 126 countries and territories between 1990 and 2019 [29].

To make it possible to compare dengue incidence in different countries, the GBD study used a series of models to estimate data. The general methodological approaches to estimate the incidence of dengue infection were described elsewhere [30]. Briefly, reported cases from published studies, governmental agencies, WHO and the Pan American Health Organization, etc., were reviewed to estimate the incidence of dengue infection. The under-reporting adjustment factors were estimated by using a regularized and trimmed meta-regression-Bayesian (MR-BRT) approach. The uncertainty from the MR-BRT meta-regression was applied to the age- and sex-specific adjustment. Once the data were adjusted for under-reporting, a hybrid approach was used to generate incidence estimates by using two models: (1) a space-time Gaussian process regression and (2) negative binomial regression using fixed effects to model all incidence [30].

\section{Regions and Demographics}

A total of 126 countries or territories reported dengue episodes among all 204 countries or territories during 1990 to 2019. These 126 countries or territories were classified into five Socio-demographic Index (SDI) regions, including low, low-middle, middle, high-middle and high SDI regions. The SDI was developed by GBD researchers and is a composite indicator of total fertility rate of those < 25 years old, years of education of those aged $\geq$ 15 years and lag distributed income per capita [31]. Meanwhile, 126 countries or territories were also separated into 17 regions based on their epidemiological homogeneity and geographical contiguity, including high-income areas of Asia Pacific, Central Asia and others in the GBD study [32]. In this study, we selected the following five age groups to analyze: < 
5 years, 5-14 years, $15-49$ years, 50-69 years and $\geq 70$ years.

\section{Statistical Analysis}

The epidemic status of dengue infection was shown by using the absolute number of dengue episodes with 95\% uncertainty intervals (UIs) and age-standardized incidence rate (ASR) with 95\% UIs. Absolute number of dengue episodes reflected the actual occurrence of dengue infection in each country or territory. Considering the importance of standardization when comparing populations with different age structures or the same population over time, we extracted the ASRs, which were calculated by applying the age-specific rates for each location, sex and year to a GBD World Standard Population [33].

We calculated the relative changes in dengue episodes from 1990 to 2019, which were defined as $\frac{\text { episodes }_{2019}-\text { episodes }_{1990}}{\text { episodes }_{1990}} \times 100 \%$ [34]. The estimated annual percentage change (EAPC) is a summary and widely used measure of the ASR trend over a specified time interval. A regression line was fitted to the natural logarithm of the ASR, that is $y=\alpha+\beta x+\varepsilon$, where $y=\ln$ (ASR) and $x=$ calendar year. EAPC (95\% CIs) was calculated as $100 \times\left(e^{\beta}-1\right)$ to measure the temporal trend of ASRs in our study [33]. If the EAPC estimation and its 95\% CIs were both $>0$ (or both $<0$ ), the ASR had an increasing trend (or a decreasing trend) [33]. Because there was a peak of ASR in 2011 globally (Figure S1), we choose 2011 as the cut-off time point and calculated the EAPC estimation of 1990-2011 and 2011-2019 two-time intervals to represent the details of changes. All statistics were performed using R 4.0.

\section{Compliance with Ethics Guidelines}

This article does not contain any new studies with human or animal subjects performed by any of the authors.

\section{RESULTS}

\section{Global and National Trends in Incidence of Dengue Infection}

One hundred twenty-six countries or territories had dengue infection records according to GBD 2019. The global number of dengue episodes increased by $85.47 \%$ from 30.67 million in 1990 to 56.88 million in 2019 (Table 1). The number of dengue episodes increased in most countries or territories except Cuba, Thailand and the US Virgin Islands, among others (Table S1 and Fig. 1). The number of dengue episodes in India (27.99 million) and China (6.10 million) accounted for almost 60\% (34.09 million/56.88 million) of the total dengue episodes in 2019 (Table S1). Furthermore, the number of dengue episodes increased by $291.22 \%$ in China from 1990 to 2019.

The ASR of dengue infection is distributed heterogeneously worldwide, and on 2019 the top three countries or territories with the highest ASRs were Niue (8749.54 per 100,000), Northern Mariana Islands (8687.22 per 100,000$)$ and Kiribati (8050.59 per 100,000) (Table S1). Globally, the ASR increased by an average of $1.70 \%$ (95\% CI 1.62\%-1.78\%) per year from 557.15 per 100,000 in 1990 to 767.93 per 100,000 in 2011; subsequently, it decreased by an average of $0.41 \%$ (95\% CI $0.20 \%-0.62 \%)$ per year from 767.93 per 100,000 in 2011 to 740.38 per 100,000 in 2019 (Table 1). Twentythree countries or territories had an increasing trend of ASRs during 2011 to 2019, most of which were in Oceania (Papua New Guinea, Fiji, Solomon Islands, etc.) (Table S1 and Figure S2).

\section{Difference in Incidence of Dengue Infection in Five SDI Regions}

For SDI regions, dengue episodes increased remarkably in high-middle (181.23\%), followed by high (109.41\%), middle $(101.70 \%)$, low (93.96\%) and low-middle (53.71\%) SDI regions (Table 1). Both the largest number of dengue episodes (23.11 million) and the highest ASR $(1317.83$ per 100,000$)$ were observed in lowmiddle SDI regions in 2019 (Table 1). The ASR 


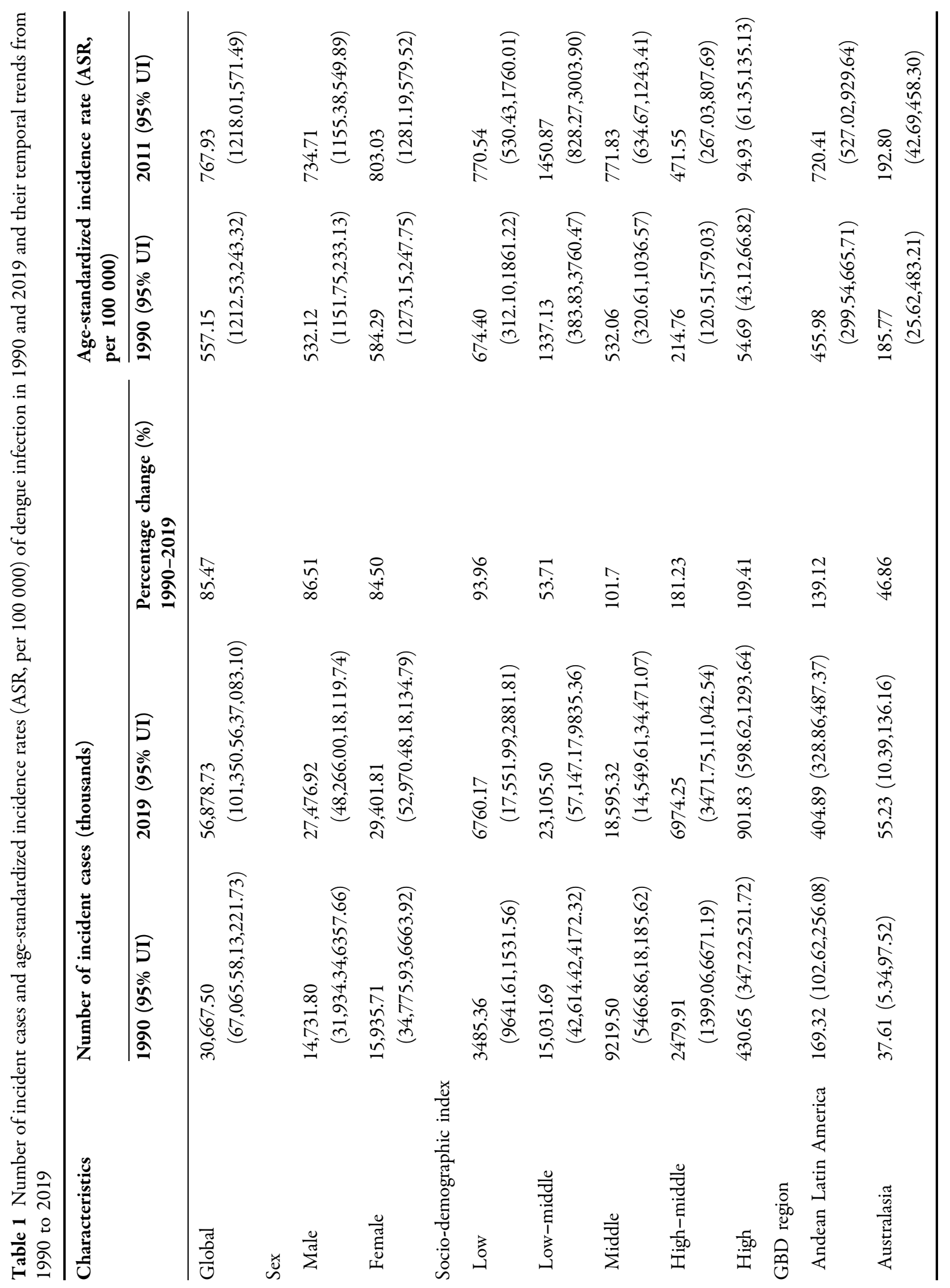




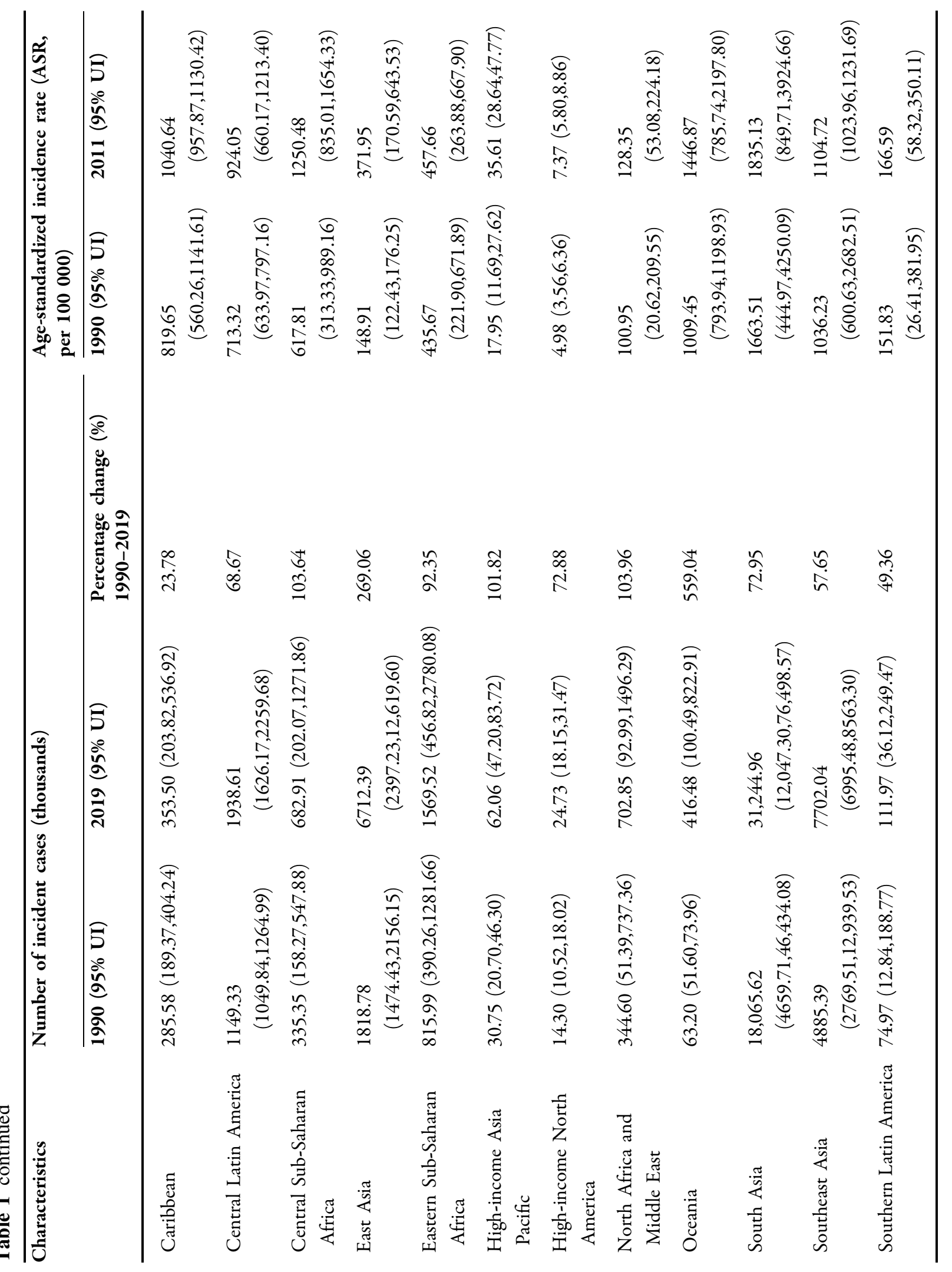




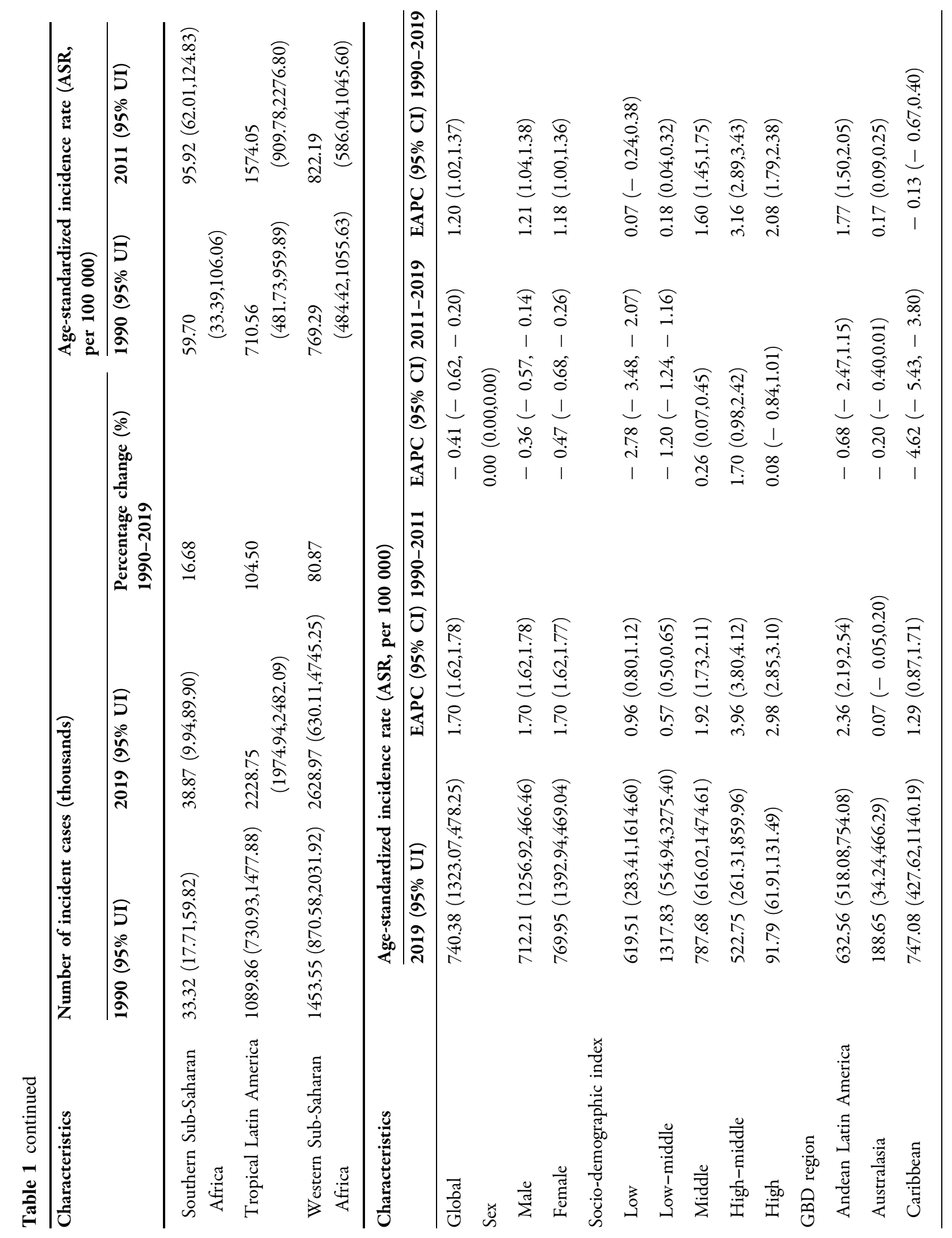




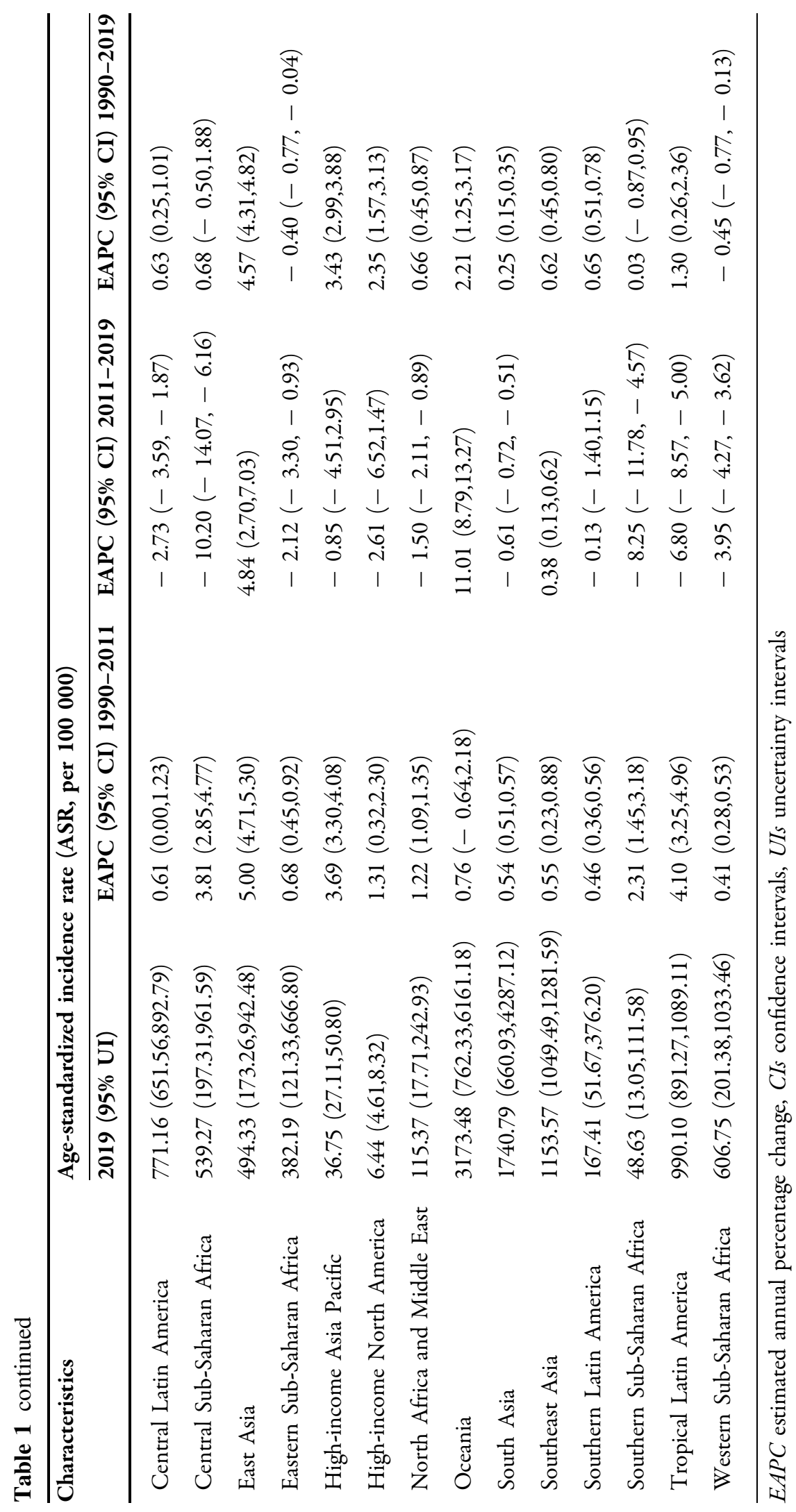



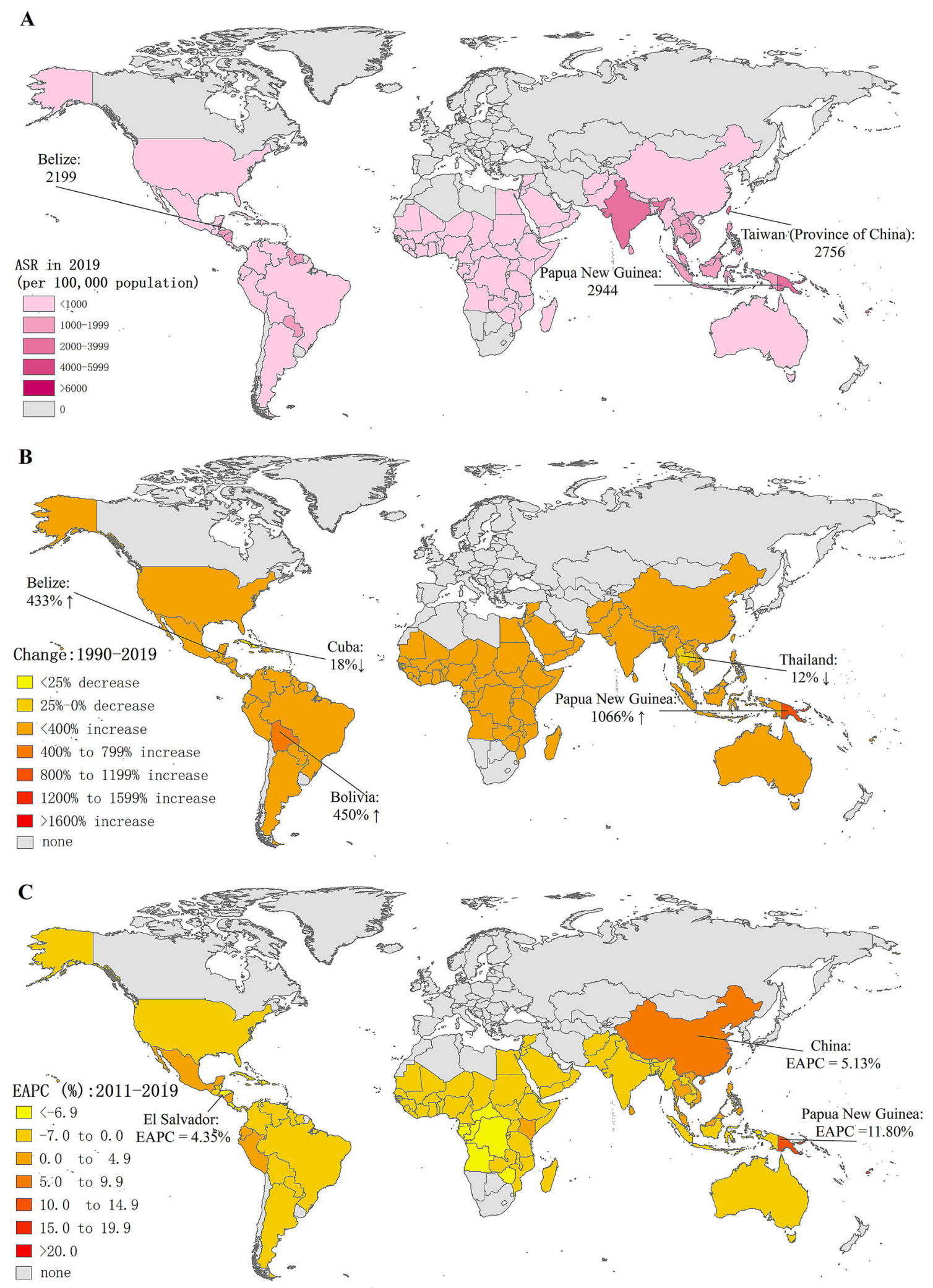
4Fig. 1 Global trends in the incidence of dengue infection in 126 countries and territories. A ASRs of dengue infection in 2019; B changes in the number of dengue episodes between 1990 and 2019; C EAPCs of dengue ASRs from 2011 to 2019. ASR, age-standardized incidence rate; $E A P C$ estimated annual percentage change

increased in all SDI regions during 1990 to 2011, with the highest ASR in high-middle SDI regions (EAPC 3.96, 95\% CI 3.80-4.12). During 2011 to 2019 , the ASR decreased in low and lowmiddle SDI regions, while the middle and highmiddle regions still had an increasing trend of ASR (Table 1 and Fig. 2).

For all SDI regions, the growing number of dengue episodes was due to the gradual increase in the number of cases in all age groups (Figure S3). The peak age of global dengue incidence rates was in adolescents and young adults
(10-25 years), followed by young children (5-10 years) and middle-aged adults (25-49 years), and rebounded slightly in the elderly ( $>$ 65 years) in 2019 (Fig. 4). Moreover, the dengue incidence rates were highest in the elderly ( $>$ 65 years), followed by adolescents and young adults (10-25 years) and young children (5-10 years) in low and low-middle SDI regions (Fig. 3).

\section{Difference in Incidence of Dengue Infection in 17 GBD Regions}

Seventeen GBD regions had dengue infection records according to GBD 2019. The number of dengue episodes was highest in South Asia (7.70 million in 2019), followed by East Asia and Southeast Asia (Table 1). The greatest growth in the number of dengue episodes was in Oceania by $559.04 \%$ from 63.20 thousand in 1990 to

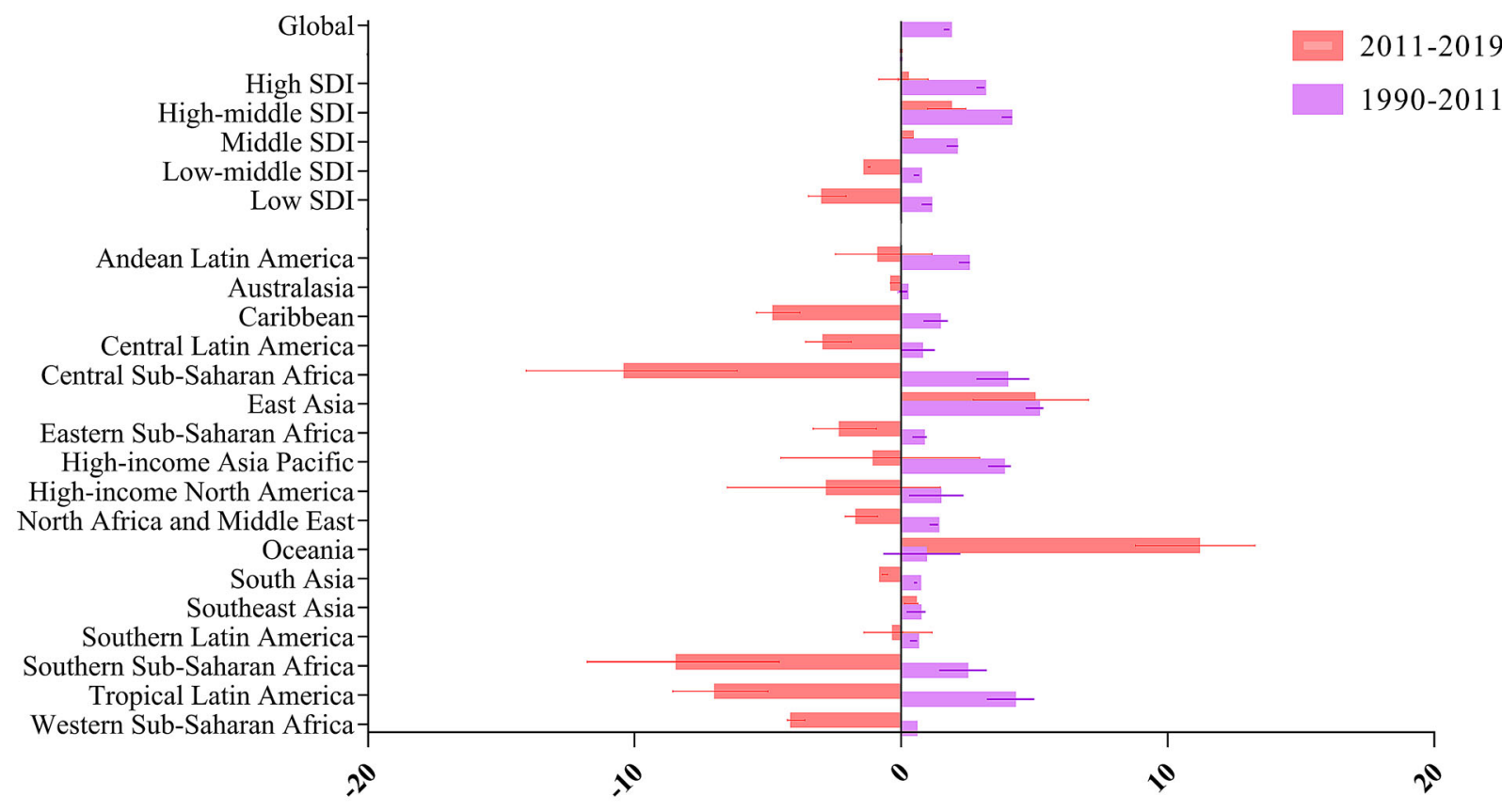

EAPC $(95 \% \mathrm{CI})$

Fig. 2 EAPCs of dengue ASRs from 1990 to 2011 and 2011 to 2019 by region. EAPCs: estimated annual percentage changes; $A S R$ age-standardized incidence rate, $C I$ confidence interval, $S D I$ sociodemographic index 

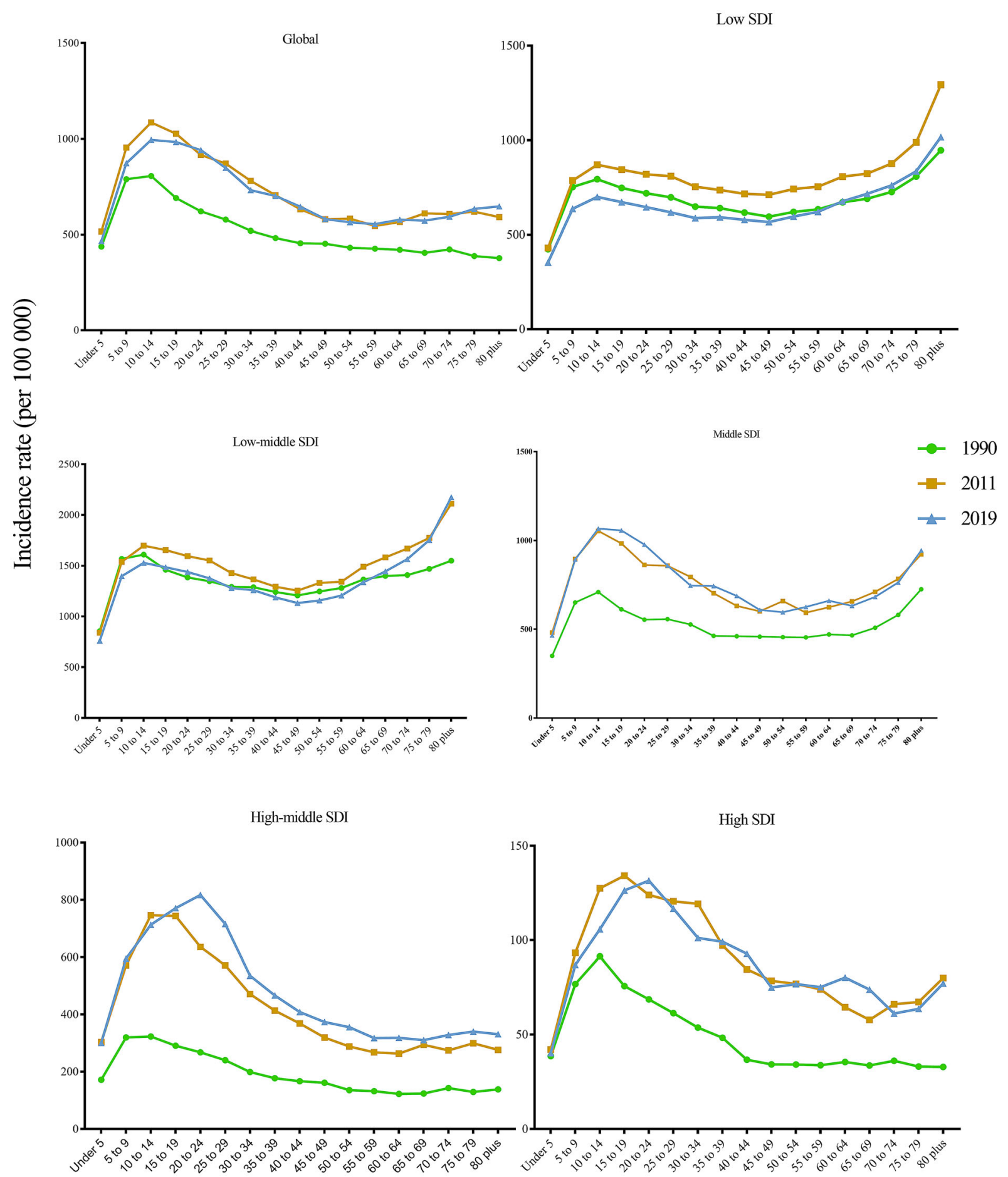

Fig. 3 Incidence rate of dengue by age group, and by SDI region, from 1990 to 2019. SDI socio-demographic index

416.48 thousand in 2019, and the number of dengue episodes in East Asia has more than doubled during the past 3 decades. Additionally, great growth was seen in the number of 
dengue episodes in Andean Latin America (139.12\%) followed by Tropical Latin America (104.50\%).

Oceania suffered the severest threat of dengue infection with the ASR $>3173.48$ per 100,000 in 2019, and South Asia and Southeast Asia also suffered a severe dengue threat with the ASR > 1000 per 100,000 in 2019 (Table 1). In addition, in the Americas, in 2019 the ASR was highest in Tropical Latin America (990.10 per 100,000) followed by Central Latin America (771.16 per 100,000) and Andean Latin America (632.56 per 100,000$)$. From 2011 to 2019 , nearly 14 GBD regions had a decreasing ASR trend (South Asia, Tropical Latin America and Central Latin America, etc.), but Oceania had the highest increasing trend (EAPC 11.01, 95\% CI 8.79-13.27), followed by East Asia (EAPC 4.84, 95\% CI 2.70-7.03) and Southeast Asia (EAPC 0.38, 95\% CI 0.13-0.62) (Table 1).

The proportions of dengue episodes by age group at the GBD regional level in 1990, 2011 and 2019 are presented in Fig. 4. Globally, > $80 \%$ of the episodes were in people $<50$ years old in 2019. However, $>10 \%$ of episodes were among the elderly ( $\geq 70$ years) in high-income regions including high-income North America and Australasia regions in 2019. Notably, nearly $20 \%$ of those affected were elderly ( $\geq 70$ years) in high-income North America (Fig. 4). The proportions of dengue episodes in the $\geq 70$ year age group increased in 2019 (using the baseline in 1990 or 2011) among most GBD regions (Fig. 4).

\section{DISCUSSION}

To our knowledge, this is the first study to assess the global landscape, long-term trends and regional differences in the incidence of dengue infection by using the GBD 2019 study. Although the global ASR of dengue infection showed an increasing trend from 1990 to 2011, a decreasing trend was observed from 2011 to 2019. Conversely, an increasing trend of ASR was reported in Oceania, East Asia and Southeast Asia. Besides, middle and high-middle SDI regions also had an increasing ASR trend during the 2011-2019 period. This finding alerted us that the fight against dengue is still challenging. The dengue incidence rates were highest in adolescents and young adults globally. Differently, it was highest in elderly people ( $>$ 65 years) in low and low-middle SDI regions, and the proportions of dengue episodes in the $>$ 70-year age group increased in 2019 (using the baseline in 1990 or 2011) among most GBD regions. Meanwhile, for the Asias, the number of dengue episodes significantly expanded in 2019 in South Asia, East Asia and Southeast Asia compared with 1990. Although regions of the
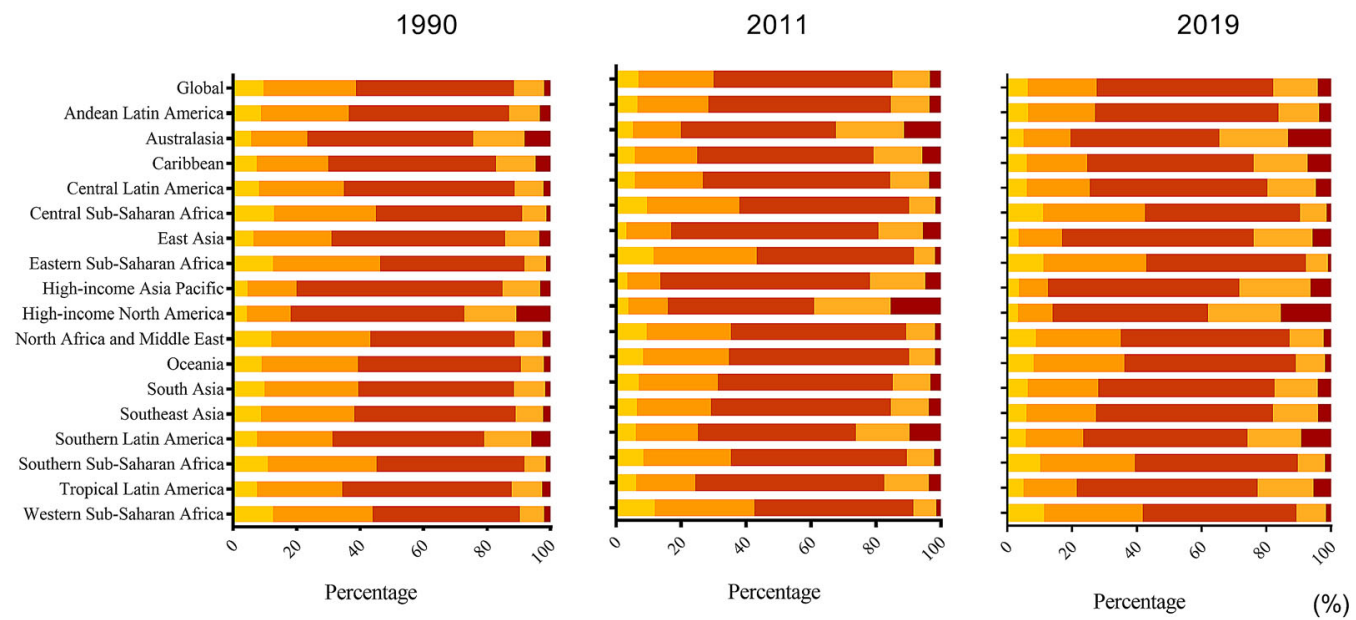

Fig. 4 Age group distribution of dengue episodes by GBD region in 1990, 2011 and 2019 
Americas had a decreasing ASR trend from 2011 to 2019 , the number of dengue episodes also significantly expanded in 2019 in Andean Latin America and Tropical Latin America compared with 1990. In addition, Tropical Latin America, Central Latin America and Andean Latin America also had relatively higher ASR in 2019 in the Americas. Facing these complex situations of this long-neglected virus at the level of different regions, there still is a need for prevention of dengue infection, and more efforts should be made to eliminate it.

Our results showed that the South Asia, East Asia and Southeast Asia regions suffered the most severe threat of dengue infection. In South Asia, India, Bangladesh, Pakistan, Maldives and Nepal are the main countries endemic for dengue fever [5, 35]. In East Asia, China [6, 36], Japan [37] and Korea [24], all had dengue episodes. Except for Brunei in Southeast Asia, Singapore, Malaysia, Indonesia, Myanmar, Thailand, Laos, Cambodia, Vietnam and the Philippines have reported epidemic dengue in recent years [38, 39]. Among these regions, the dengue episodes in India and China accounted nearly $60 \%$ of all global episodes.

Notably, East Asia and Southeast Asia not only had the highest dengue burden but also had an increasing ASR compared to the global falling trend of ASR from 2011 to 2019. For East Asia, China is one of the dengue endemic countries and faces the great challenges of dengue infection in nearly 10 years. The number of dengue episodes increased by $291.22 \%$ in China from 1990 to 2019, and the ASR had an increasing trend in 2011-2019. The imported cases (from Southeast Asia and Western Pacific countries) might contribute to dengue infection in China [40-42]. With the increasing number of imported cases, local dengue infection outbreaks have occurred more frequently [42]. Bionomics and insecticide resistance of Aedes albopictus [43, 44], climate factors and the East Asian summer monsoon may also drive the increasing ASR of dengue infection in China [45]. In response to this situation, integrated vector management should be implemented including new innovative vector control activities and traditional vector control measures $[23,27,46]$. An early start of response could effectively mitigate the dengue burden by reducing the mosquito vector density [47]. Vector-control measures seem to be insufficient; travel surveillance and management should also be strengthened to reduce the possibilities of dengue outbreaks in East Asia. People should be informed about dengue prevention when planning a journey to endemic regions during peak dengue transmission periods [48]. For Southeast Asia, urban settlements with high population densities, lack of public perception and limited infrastructure and resources may have accelerated outbreaks in recent years [48]. Similarly, vector-control measures are crucial; furthermore, planned urbanization and enhanced infrastructure and resources should be developed [48, 49]. People should participate in mosquito control, such as using nets especially early in the morning and before dusk in the evening [27], clean up discarded tires and watercollecting waste $[23,50,51]$ and use insecticidetreated nets, screens and walls [52].

Although most regions of the Americas had a decreasing ASR trend from 2011 to 2019, Andean Latin America and Tropical Latin America had relatively higher growth of dengue episodes from 1990 to 2019. Besides, Tropical Latin America, Central Latin America and Andean Latin America also had non-negligible ASRs in 2019. The epidemiological data from the Ministries of Health showed that 1,118,464 dengue episodes were reported in Central America and the Dominican Republic from 2005 to 2014 [53]. In 2013, more than 2 million dengue episodes were reported by countries in the Pan American Health Organization [54]. Therefore, considering the above situation, the epidemic of dengue also needs to be a focus in the Americas, especially in Latin America. Factors which contribute to increasing dengue episodes were the same as in most other regions globally including unprecedented population growth, unplanned and uncontrolled urbanization and poverty, etc. [54]. Therefore, the above-mentioned strategies including strengthening national surveillance systems, integrated vector management and personal behavioral interventions are all needed in the Americas. Additionally, a future dengue vaccine could be 
integrated into a management strategy for dengue prevention and control [54].

We also found the middle and high-middle SDI regions had an increasing trend of ASR during the 2011-2019 period, which was unexpected. WHO proposed a global strategy for dengue prevention and control in 2012-2020 to achieve a mortality from dengue $<0.1 \%$ and to reduce morbidity by $50 \%$ by 2020 [27]. Many countries or territories which had an increasing ASR trend in the middle and high-middle SDI regions are in Oceania including Papua New Guinea, Fiji, Solomon Islands and others. First, these countries or territories have a tropical or sub-tropical climate in the South Pacific Ocean, which accelerates dengue outbreaks. Second, some people still live in poverty and are affected by dengue disproportionately in Papua New Guinea, Fiji, Solomon Islands and others [55]. Studies reported dengue could emerge as an important neglected tropical disease pathogen in these countries or territories in the coming decade, so integration of monitoring and evaluation related to dengue epidemics is needed to provide assistance in controlling the rising threat of dengue [55].

In addition, this study found that the global peak dengue incidence rate occurred in adolescents and young adults in 2019, which shows that adolescents and adults were mostly affected by dengue in most regions. This finding is in accordance with previous studies $[1,6]$. However, our study emphasized that the dengue incidence rates were highest in elderly people ( $>$ 65 years) in low and low-middle SDI regions. In addition, the proportions of dengue episodes in the $>70$-year-old age group increased in 2019 (using the baseline in 1990 or 2011) among most GBD regions. Chhong et al. reported that dengue infection was common in older adults in Bangkok Hospital of Thailand [13]. The age of dengue fever cases in Taiwan (province of China) was generally older than that in other countries [14]. Research proposed that successful vector control and surveillance programs lead to lower seroprevalence and herd immunity in adults, finally causing acquisition of disease at a later stage in life [56]. Additionally, the increased proportions of dengue episodes among older people may be linked to the aging trend globally. Higher incidence rates and proportions of dengue infection among older people could potentially result in increases in dengue case fatality rates, given the higher risk of mortality in older people [57]. Therefore, we suggest that management of dengue infection should be resolved differently and seriously among older people. Endemic countries with limited resources or vaccine supply should have rational means to decide which segments of the population to protect [27]. Moreover, the safety and efficacy of the vaccine in the adult and elderly population need to be determined [56]. Unfortunately, related studies on the management of dengue infection in the elderly are scarce.

We extracted the data from the GBD 2019 and added a comprehensive understanding of the incidence of dengue infection to help develop global targeted prevention strategies. However, there still were several limitations. First, the most notable limitation of this analysis was that the accuracy and robustness of GBD estimates largely depend on the quality and quantity of data used in the modeling [33]. For countries where national systematic surveillance and population-based studies of dengue were lacking or insufficient, the estimates of dengue incidence might be a margin of bias. Additionally, since the GBD concentrated information is derived from national epidemiological surveillance systems, which are highly heterogeneous because of the quality of clinical and laboratory diagnostics, laboratory quality and reporting standards among different countries, the plausibility of the results would be influenced compared with observational studies or clinical trials [58-62], which should be considered part of the interpretation of heterogeneous data $[53,54]$. Second, there were no data associated with dengue genotypes, which is the key factor for the transmission and epidemiology of dengue globally. Therefore, the dengue epidemic situation for one country combined with the local dengue genotypes could help develop more exact prevention measures. 


\section{CONCLUSION}

In summary, global dengue episodes increased tremendously from 1990 to 2019. Although the global dengue ASR decreased in the last decade, it continued to increase in hyperendemic regions including Oceania, East Asia and Southeast Asia and also in the middle and highmiddle SDI regions. More attention should be paid to the elderly due to their higher dengue incidence rate in low and low-middle SDI regions and the increased proportions of dengue episodes among the elderly in most GBD regions. Generally, targeted measures for crucial regions and older populations were needed. Therefore, more studies focusing on dengue need to be carried out to achieve the goal of dengue elimination.

\section{ACKNOWLEDGEMENTS}

We appreciate the work of the Global Burden of Disease study 2019 collaborators.

Funding3. This study was funded by a grant from the National Natural Science Foundation of China (no. 71934002). The Journal's Rapid Service Fee was funded by the authors.

Authorship3. All named authors meet the International Committee of Medical Journal Editors (ICMJE) criteria for authorship for this article, take responsibility for the integrity of the work as a whole, and have given their approval for this version to be published.

Authorship Contributions3. Conceptualization: Min Liu. Data curation: Min Du. Formal analysis: Min Du. Funding acquisition: Min Liu. Methodology: Min Liu, Wenzhan Jing.. Supervision: Jue Liu, Wenzhan Jing. Validation: Wenzhan Jing., Jue Liu. Writing-original draft: Min Du. Writing_review and editing: Min Du, Wenzhan Jing., Jue Liu, Min Liu. All authors participated in data analysis, interpretation, discussion, and writing of the manuscript.
Disclosures3. Min Du, Wenzhan Jing, Jue Liu and Min Liu all have nothing to disclose.

Compliance with Ethics Guidelines3. This article does not contain any new studies with human or animal subjects performed by any of the authors.

Data Availability3. The datasets generated during and/or analyzed during the current study are available in the GBD repository, [http://ghdx.healthdata.org/gbd-results-tool].

Open Access3. This article is licensed under a Creative Commons Attribution-NonCommercial 4.0 International License, which permits any non-commercial use, sharing, adaptation, distribution and reproduction in any medium or format, as long as you give appropriate credit to the original author(s) and the source, provide a link to the Creative Commons licence, and indicate if changes were made. The images or other third party material in this article are included in the article's Creative Commons licence, unless indicated otherwise in a credit line to the material. If material is not included in the article's Creative Commons licence and your intended use is not permitted by statutory regulation or exceeds the permitted use, you will need to obtain permission directly from the copyright holder. To view a copy of this licence, visit http:// creativecommons.org/licenses/by-nc/4.0/.

\section{REFERENCES}

1. Wilder-Smith A, Ooi EE, Horstick O, Wills B. Dengue Lancet. 2019;393(10169):350-63. https://doi. org/10.1016/S0140-6736(18)32560-1.

2. Shepard DS, Undurraga EA, Halasa YA, Stanaway JD. The global economic burden of dengue: a systematic analysis. Lancet Infect Dis. 2016;16(8): 935-41. https://doi.org/10.1016/S14733099(16)00146-8.

3. World Health Organization. Dengue and severe dengue. 2020; https://www.who.int/health-topics/ dengue-and-severe-dengue\#tab=tab_1. 
4. Liebig J, Jansen C, Paini D, Gardner L, Jurdak R. A global model for predicting the arrival of imported dengue infections. PLoS One. 2019;14(12): e0225193. https://doi.org/10.1371/journal.pone. 0225193 (Published 2019 Dec 4).

5. Khadka S, Proshad R, Thapa A, Acharya KP, Kormoker T. Wolbachia: a possible weapon for controlling dengue in Nepal. Trop Med Health. 2020;48:50. https://doi.org/10.1186/s41182-02000237-4 (Published 2020 Jun 22).

6. Zhang H, Mehmood K, Chang YF, Zhao Y, Lin W, Chang Z. Increase in cases of dengue in China, 2004-2016: A retrospective observational study. Travel Med Infect Dis. 2020. https://doi.org/10. 1016/j.tmaid.2020.101674.

7. Lai WT, Chen $\mathrm{CH}$, Hung H, Chen RB, Shete S, Wu CC. Recognizing spatial and temporal clustering patterns of dengue outbreaks in Taiwan. BMC Infect Dis. 2018;18(1):256. https://doi.org/10.1186/ s12879-018-3159-9 (Published 2018 Jun 4).

8. Messina JP, Brady OJ, Golding N, Kraemer MUG, Wint GRW, Ray SE, et al. The current and future global distribution and population at risk of dengue. Nat Microbiol. 2019;4(9):1508-15. https://doi. org/10.1038/s41564-019-0476-8.

9. Sim S, Hibberd ML. Genomic approaches for understanding dengue: insights from the virus, vector, and host. Genome Biol. 2016;17:38. https:// doi.org/10.1186/s13059-016-0907-2 (Published 2016 Mar 2).

10. Mustafa MS, Rasotgi V, Jain S, Gupta V. Discovery of fifth serotype of dengue virus (DENV-5): a new public health dilemma in dengue control. Med J Armed Forces India. 2015;71(1):67-70. https://doi. org/10.1016/j.mjafi.2014.09.011.

11. Freeman MC, Coyne CB, Green M, Williams JV, Silva LA. Emerging arboviruses and implications for pediatric transplantation: a review. Pediatr Transpl. 2019;23(1):e13303. https://doi.org/10.1111/petr. 13303.

12. Otu A, Ebenso B, Etokidem A, Chukwuekezie O. Dengue fever-an update review and implications for Nigeria, and similar countries. Afr Health Sci. 2019;19(2):2000-7. https://doi.org/10.4314/ahs. v19i2.23.

13. Chhong LN, Poovorawan K, Hanboonkunupakarn B, Phumratanaprapin W, Soonthornworasiri N, Kittitrakul C, et al. Prevalence and clinical manifestations of dengue in older patients in Bangkok Hospital for Tropical Diseases, Thailand. Trans R Soc Trop Med Hyg. 2020;114(9):674-81. https:// doi.org/10.1093/trstmh/traa043.
14. Hsieh CC, Cia CT, Lee JC, Sung JM, Lee NY, Chen PL, et al. A cohort study of adult patients with severe Dengue in Taiwanese intensive care units: the elderly and APTT prolongation matter for prognosis. PLoS Negl Trop Dis. 2017;11(1): e0005270. https://doi.org/10.1371/journal.pntd. 0005270 (Published 2017 Jan 6).

15. Wang WH, Chen HJ, Lin CY, Assavalapsakul W, Wang SF. Imported dengue fever and climatic variation are important determinants facilitating dengue epidemics in Southern Taiwan. J Infect. 2020;80(1):121-42. https://doi.org/10.1016/j.jinf. 2019.08.010.

16. Mahmood S, Irshad A, Nasir JM, Sharif F, Farooqi SH. Spatiotemporal analysis of dengue outbreaks in Samanabad town, Lahore metropolitan area, using geospatial techniques. Environ Monit Assess. 2019;191(2):55. https://doi.org/10.1007/s10661018-7162-9 (Published 2019 Jan 7).

17. Costa SDSB, Branco MDRFC, Aquino Junior J, Silva MDSD, Costa FRVD, Santos AVDD, et al. Spatial analysis of probable cases of dengue fever, chikungunya fever and zika virus infections in Maranhao State, Brazil. Rev Inst Med Trop Sao Paulo. 2018;60: e62. https://doi.org/10.1590/S1678-99462018600 62 [published correction appears in Rev Inst Med Trop Sao Paulo. 2018 Dec 10;60:e62err; Published 2018 Oct 25].

18. Da Conceição Araújo $\mathrm{D}$, Dos Santos $\mathrm{AD}$, Lima SVMA, Vaez AC, Cunha JO, Conceição Gomes Machado de Araújo K. Determining the association between dengue and social inequality factors in north-eastern Brazil: a spatial modelling. Geospat Health. 2020. https://doi.org/10.4081/gh.2020.854 (Published 2020 Jun 17).

19. Cattarino L, Rodriguez-Barraquer I, Imai N, Cummings DAT, Ferguson NM. Mapping global variation in dengue transmission intensity. Sci Transl Med. 2020;12(528):eaax4144. https://doi.org/10. 1126/scitranslmed.aax4144.

20. Wellekens K, Betrains A, De Munter P, Peetermans W. Dengue: current state one year before WHO 2010-2020 goals. Acta Clin Belg. 2020. https://doi. org/10.1080/17843286.2020.1837576 [published online ahead of print, 2020 Oct 22].

21. Lourenço J, Recker M. Dengue serotype immuneinteractions and their consequences for vaccine impact predictions. Epidemics. 2016;16:40-8. https://doi.org/10.1016/j.epidem.2016.05.003.

22. Robinson ML, Durbin AP. Dengue vaccines: implications for dengue control. Curr Opin Infect Dis. 2017;30(5):449-54. https://doi.org/10.1097/QCO. 0000000000000394 . 
23. Salazar F, Angeles J, Sy AK, et al. Efficacy of the In2Care ${ }^{\circledR}$ auto-dissemination device for reducing dengue transmission: study protocol for a parallel, two-armed cluster randomised trial in the Philippines. Trials. 2019;20(1):269. https://doi.org/10. 1186/s13063-019-3376-6 (Published 2019 May 14).

24. Lee H, Kim JE, Lee S, Lee CH. Potential effects of climate change on dengue transmission dynamics in Korea. PLoS One. 2018;13(6):e0199205. https:// doi.org/10.1371/journal.pone.0199205 (Published 2018 Jun 28).

25. Coldbeck-Shackley RC, Eyre NS, Beard MR. The Molecular Interactions of ZIKV and DENV with the Type-I IFN Response. Vaccines (Basel). 2020;8(3): 530. https://doi.org/10.3390/vaccines 8030530 (Published 2020 Sep 14).

26. Gyawali N, Bradbury RS, Taylor-Robinson AW. The epidemiology of dengue infection: Harnessing past experience and current knowledge to support implementation of future control strategies. J Vector Borne Dis. 2016;53(4):293-304.

27. Global strategy for dengue prevention and control 2012-2020. WHO Geneva: World Health Organization; 2012. http://apps.who.int/iris/bitstream/ 10665/75303/1/9789241504034_eng.pdf. Accessed 8 Jul 2016.

28. Stanaway JD, Shepard DS, Undurraga EA, Halasa YA, Coffeng LE, Brady OJ, et al. The global burden of dengue: an analysis from the Global Burden of Disease Study 2013. Lancet Infect Dis. 2016;16(6): 712-23. 3099(16)00026-8.

29. Global Burden of Disease Collaborative Network. Global Burden of Disease Study 2017 (GBD 2017) Results. Seattle, United States: Institute for Health Metrics and Evaluation (IHME); 2018. https://ghdx. healt hdata.org/gbd-resul ts-tool. Accessed $10 \mathrm{Sep}$ 2019.

30. GBD 2019 Diseases and Injuries Collaborators. Global burden of 369 diseases and injuries in 204 countries and territories, 1990-2019: a systematic analysis for the Global Burden of Disease Study 2019. Lancet. 2020;396(10258):1204-1222. https:// doi.org/10.1016/S0140-6736(20)30925-9 [published correction appears in Lancet. 2020 Nov 14;396(10262):1562].

31. Global Burden of Disease Collaborative Network. Global Burden of Disease Study 2019 (GBD 2019) Socio-Demographic Index (SDI) 1950-2019. 2020. http://ghdx.healthdata.org/record/ihme-data/gbd2019-socio-demographic-index-sdi-1950-2019. Accessed 3 Mar 2021.
32. Murray CJ, Ezzati M, Flaxman AD, Lim S, Lozano R, Michaud C, et al. GBD 2010: design, definitions, and metrics. Lancet. 2012;380(9859):2063-6. https://doi.org/10.1016/S0140-6736(12)61899-6.

33. Liu Z, Jiang Y, Yuan H, Fang Q, Cai N, Suo C, et al. The trends in incidence of primary liver cancer caused by specific etiologies: Results from the Global Burden of Disease Study 2016 and implications for liver cancer prevention. J Hepatol. 2019;70(4): 674-83. https://doi.org/10.1016/j.jhep.2018.12. 001.

34. Wenzhan J, Liu J, Liu M. The global trends and regional differences in incidence of HEV infection from 1990 to 2017 and implications for HEV prevention. Liv Int. 2021;41(1):58-69. https://doi.org/ 10.1111/liv.14686.

35. Mishra AC, Arankalle VA, Gadhave SA, Mahadik $\mathrm{PH}$, Shrivastava S, Bhutkar M, et al. Stratified seroprevalence revealed overall high disease burden of dengue but suboptimal immunity in younger age groups in Pune, India. PLoS Negl Trop Dis. 2018;12(8):e0006657. https://doi.org/10.1371/ journal.pntd.0006657 (Published 2018 Aug 6).

36. Mu D, Cui JZ, Yin WW, Li Y, Chen QL. Zhonghua Liu Xing Bing Xue Za Zhi. 2020;41(5):685-9. https://doi.org/10.3760/cma.j.cn112338-2019071500523.

37. Saijo M. Yakugaku Zasshi. 2020;140(7):895-9. https://doi.org/10.1248/yakushi.19-00255-2.

38. Liang $\mathrm{Y}$, Ahmad Mohiddin MN, Bahauddin R, Hidayatul FO, Nazni WA, Lee HL, et al. Modelling the effect of a novel autodissemination trap on the spread of Dengue in Shah Alam and Malaysia. Comput Math Methods Med. 2019;2019:1923479. https://doi.org/10.1155/2019/1923479 (Published 2019 Aug 4).

39. Dyer O. Dengue: Philippines declares national epidemic as cases surge across South East Asia. BMJ. 2019;366:15098. https://doi.org/10.1136/bmj.15098 (Published 2019 Aug 8).

40. Yao MX, Wu SZ, Wang GL, Wang XJ, Fan WJ, Zhang WG, et al. Imported dengue serotype 1 outbreak in a non-endemic region, China, 2017: a molecular and seroepidemiological study. J Infect. 2020;81(2):304-10. https://doi.org/10.1016/j.jinf. 2020.06.016.

41. Yan H, Ding Z, Yan J, Yao W, Pan J, Yang Z, et al. Epidemiological characterization of the 2017 Dengue outbreak in Zhejiang, China and molecular characterization of the viruses. Front Cell Infect Microbiol. 2018;8:216. https://doi.org/10.3389/ fcimb.2018.00216 (Published 2018 Jul 4). 
42. Wang JZ, You LB, Kan NP, Lin Q, Weng YW, Zheng KC. Frequent import and multiple sources of dengue fever have changed the epidemic situation of the disease in Fujian Province, China. Biomed Environ Sci. 2020;33(2):123-32. https://doi.org/10. 3967/bes2020.016.

43. Liu H, Liu L, Cheng P, Yang L, Chen J, Lu Y, et al. Bionomics and insecticide resistance of Aedes albopictus in Shandong, a high latitude and highrisk dengue transmission area in China. Parasit Vectors. 2020;13(1):11. https://doi.org/10.1186/ s13071-020-3880-2 (Published 2020 Jan 9).

44. Li Y, Zhou G, Zhong D, Wang X, HemmingSchroeder E, David RE, et al. Widespread multiple insecticide resistance in the major dengue vector Aedes albopictus in Hainan Province, China. Pest Manag Sci. 2021;77(4):1945-53. https://doi.org/10. $1002 /$ ps.6222.

45. Liu K, Hou X, Ren Z, Lowe R, Wang Y, Li R, et al. Climate factors and the East Asian summer monsoon may drive large outbreaks of dengue in China. Environ Res. 2020;183:109190. https://doi.org/10. 1016/j.envres.2020.109190.

46. Kamtchum-Tatuene J, Makepeace BL, Benjamin L, Baylis M, Solomon T. The potential role of Wolbachia in controlling the transmission of emerging human arboviral infections. Curr Opin Infect Dis. 2017;30(1):108-16. https://doi.org/10.1097/QCO. 0000000000000342 .

47. Li L, Liu WH, Zhang ZB, Liu Y, Chen XG, Luo L, et al. The effectiveness of early start of Grade III response to dengue in Guangzhou, China: A population-based interrupted time-series study. PLoS Negl Trop Dis. 2020;14(8):e0008541. https://doi. org/10.1371/journal.pntd.0008541

(Published 2020 Aug 7).

48. Pathak VK, Mohan M. A notorious vector-borne disease: Dengue fever, its evolution as public health threat. J Family Med Prim Care. 2019;8(10): 3125-3129. https://doi.org/10.4103/jfmpc.jfmpc_ 716_19 (Published 2019 Oct 31).

49. Godaert L, Dramé M, Roubaud-Baudron C. Emerging viruses in older population Chikungunya, West Nile fever and Dengue. Aging Clin Exp Res. 2021;33(3):723-7. https://doi.org/10.1007/s40520019-01389-y.

50. Mai VQ, Mai TTX, Tam NLM, Nghia LT, Komada K, Murakami H. Prevalence and risk factors of Dengue Infection in Khanh Hoa Province, Viet Nam: a stratified cluster sampling survey. J Epidemiol. 2018;28(12):488-97. https://doi.org/10.2188/jea. JE20170090.
51. Sarti E, Cox H, Besada-Lombana S, Tapia-Maruri L. Dengue Awareness in Latin American Populations: A Questionnaire Study. Infect Dis Ther. 2015;4(2): 199-211. https://doi.org/10.1007/s40121-0150068-8.

52. Golding N, Wilson AL, Moyes CL, et al. Integrating vector control across diseases. BMC Med. 2015;13: 249. https://doi.org/10.1186/s12916-015-0491-4 (Published 2015 Oct 1).

53. Ávila-Agüero ML, Camacho-Badilla $\mathrm{K}$, Brea-DelCastillo J, Cerezo L, Dueñas L, Luque M, et al. Epidemiología del dengue en Centroamérica y República Dominicana [Epidemiology of dengue in Central America and the Dominican Republic]. Rev Chilena Infectol. 2019;36(6):698-706. https://doi. org/10.4067/S0716-10182019000600698.

54. Zambrano B, San Martin JL. Epidemiology of Dengue in Latin America. J Pediatric Infect Dis Soc. 2014;3(3):181-2. https://doi.org/10.1093/jpids/ piu071.

55. Kline K, McCarthy JS, Pearson M, Loukas A, Hotez PJ. Neglected tropical diseases of Oceania: review of their prevalence, distribution, and opportunities for control. PLoS Negl Trop Dis. 2013;7(1):e1755. https://doi.org/10.1371/journal.pntd.0001755.

56. Lin RJ, Lee TH, Leo YS. Dengue in the elderly: a review. Expert Rev Anti Infect Ther. 2017;15(8): 729-35. https://doi.org/10.1080/14787210.2017. 1358610 .

57. Hsieh CC, Hong MY, Ho TS, Liu CC, Perng GC, Chuang CC. Dynamic changes of soluble ST2 levels predicted fatality and were involved in coagulopathy in dengue fever in the elderly. PLoS Negl Trop Dis. 2019;13(12):e0007974. https://doi.org/10. 1371/journal.pntd.0007974 (Published 2019 Dec 26).

58. Vitale M, Lupone CD, Kenneson-Adams A, Ochoa RJ, Ordoñez T, Beltran-Ayala E, et al. A comparison of passive surveillance and active cluster-based surveillance for dengue fever in southern coastal Ecuador. BMC Public Health. 2020;20(1):1065. https://doi.org/10.1186/s12889-020-09168-5 (Published 2020 Jul 6).

59. Hoen AG, Keller M, Verma AD, Buckeridge DL, Brownstein JS. Electronic event-based surveillance for monitoring dengue. Latin America Emerg Infect Dis. 2012;18(7):1147-50. https://doi.org/10.3201/ eid1807.120055.

60. Gupta BP, Haselbeck A, Kim JH, Marks F, Saluja T. The Dengue virus in Nepal: gaps in diagnosis and surveillance. Ann Clin Microbiol Antimicrob. 2018;17(1):32. https://doi.org/10.1186/s12941-0180284-7 (Published 2018 Jul 16). 
61. Zhang H, Li Z, Lai S, Clements AC, Wang L, Yin W, et al. Evaluation of the performance of a dengue outbreak detection tool for China. PLoS One. 2014;9(8):e106144. https://doi.org/10.1371/ journal.pone.0106144 (Published 2014 Aug 29).

62. Standish K, Kuan G, Avilés W, Balmaseda A, Harris E. High dengue case capture rate in four years of a cohort study in Nicaragua compared to national surveillance data. PLoS Negl Trop Dis. 2010;4(3): e633. https://doi.org/10.1371/journal.pntd. 0000633 (Published 2010 Mar 16).

\section{Publisher's Note}

Springer Nature remains neutral with regard to jurisdictional claims in published maps and institutional affiliations. 